Department of Primary Care and Public Health, School of Public Health, Imperial College London, London, UK

2 Paediatrics and Pregnancy Expert Group, Faculty of Pharmaceutical Medicine, Royal College of Physicians, UK

3 Section of Neonatal Medicine, Department of Primary Care and Public Health, School of Public Health Imperial College London, London, UK

Correspondence to: A Abbas-Hanif allyah.abbas@imperial.ac.uk

Cite this as: BMJ 2021;375:n2377 http://dx.doi.org/10.1136/bmj.n2377 Published: 14 October 2021

\section{Covid-19 treatments and vaccines must be evaluated in pregnancy}

\author{
Pregnant women should be included in drug and vaccine development from the outset \\ Allyah Abbas-Hanif, ${ }^{1,2}$ Neena Modi, ${ }^{3}$ Stephen Kevin Smith, ${ }^{3}$ Azeem Majeed
}

The numbers of pregnant and postpartum women in the UK admitted to hospital or intensive care because of covid-19 peaked over the summer. ${ }^{1}$ Maternal mortality has reached concerning levels in 2021, with case fatality rates rising in the US, doubling in Brazil, and almost tripling in India since the beginning of the pandemic. ${ }^{2-4}$ In Brazil, health officials even suggested avoiding pregnancy to reduce risk during the pandemic. ${ }^{5}$

Inconsistent messaging from authorities, driven by lack of trial data, has increased covid-19 vaccine hesitancy among pregnant women. This, coupled with the increased transmissibility of new variants and relaxing of social distancing restrictions, contributed to the surge in hospital admissions seen in successive waves. Concerns around the longer term effect of covid-19 post partum, including long covid, cardiovascular complications of covid-19, and widening socioeconomic disparities are also mounting. ${ }^{6}$ Despite a desperate need for treatments, pregnant women continue to be left behind.

In the long shadow of the thalidomide and diethylstilboestrol tragedies, only one drug designed for use in pregnancy, atosiban, has been licensed in four decades and only five prescription medicines (amoxicillin, labetalol, diazoxidine injection, doxylamine with pyridoxine, sodium feredetate) are licensed for non-obstetric use in pregnancy in the UK. ${ }^{7}$ A sobering 98\% of all marketed drugs have insufficient or no safety data to guide dosing during pregnancy and lactation. ${ }^{8}$ This includes all covid-19 vaccines.

Vaccination in pregnancy is not a new concept; nor are the struggles with uptake. Concerns about covid-19 vaccination, like previous vaccines, have centred around fears of side effects for the fetus, doubts regarding efficacy, and even doubts around the need for immunisation. ${ }^{9}$ These concerns have been further compounded by misinformation regarding fertility, suspicion of the swift rollout of vaccines, and the exclusion of pregnant women from preapproval trials, with levels of vaccine hesitancy highest in deprived communities and among those from ethnic minority groups. ${ }^{10}$

Vaccination is currently recommended in pregnancy based on developmental and reproductive toxicology studies in animals, a positive risk-benefit profile in women of childbearing age, and post-marketing data from vaccinated pregnant women and their infants, rather than clinical trials. ${ }^{11}$ Despite cumulative data from over 200 ooo pregnant women showing the effectiveness of vaccination, ${ }^{11} 12$ low public confidence remains. Decisions around booster programmes are still evolving, but it is likely some pregnant women will fall into the prioritised eligible groups. ${ }^{13}$ The effect of vaccine hesitancy may, worryingly, cross over to other vaccinations such as influenza. It is essential to eradicate endemic off-label prescribing in pregnancy, which excludes women and their babies from the protections afforded by the rigours of the licensing process and creates unacceptable ethical and legal dilemmas for clinicians.

\section{Routes to change}

There are clear routes to improvement. Medicines regulators in Europe, the US, and the UK have recently taken a positive step by supporting

"maternity investigation plans," which build on the successes of paediatric investigation plans (PIPs) and orphan products. ${ }^{14}$ The introduction of PIPs through European legislation in 2007, mandated that medicines developers evaluate the needs of children for all new products. Orphan designation revitalised medicines development for rare diseases, and the US approved 31 products ( $58 \%$ of all approvals) for rare diseases in 2020. ${ }^{15}$

Regulators effectively enlivened research into these areas by offering incentives alongside obligations. Creative measures included a centralised procedure for the designation of orphan medicinal products, fee waivers, and scientific support from early development through to market authorisation. Both PIPs and orphan designation offer market exclusivity extensions after authorisation, with PIPs extending market protection to drugs no longer covered by patents, if they are then exclusively developed for use in children. A similar premise could be used to repurpose marketed drugs with a known safety profile for indications in pregnancy.

Additional strategies that can be readily implemented to invigorate development of drugs for pregnancy include prioritisation of developmental and reproductive toxicology studies at the start of drug development, consideration of physiologically based pharmacokinetic modelling, and ensuring pregnancy experts are involved in clinical trial development and in trial steering and monitoring committees. Consulting women and organisations representing their interests and those of their babies is key to ensuring pregnancy is not routinely used to exclude people from trials without clear scientific grounds.

These measures would encourage medicine developers in expanding fields, such as biological medicines, to include pregnant women from the start of the process. Monoclonal antibodies, for example, are likely to be strong therapeutic candidates for use during pregnancy among people with target conditions, potentially including covid-19. The strong antigen affinity of monoclonal antibodies makes them 
highly effective with minimal off-target activity, and placental transfer is likely to be limited, particularly during organogenesis. ${ }^{16}$

The covid-19 pandemic is radically changing the clinical trials landscape, catalysing collaborative drug development between academics, industry, and regulators, and accelerating implementation of research findings. Equity and inclusion are essential to scientific advancement, and the benefits of innovation and drug discovery should safely reach everyone. We urge regulators and governments to implement these strategies for pregnant women and their babies, who for so long have been left behind in medicines and vaccines development. An urgent shift in policy and investment is required to ensure that inclusion becomes the norm for pregnancy in development plans, unless otherwise fully justified. This will help counter vaccine hesitancy and improve confidence in the use of new treatments, leading to better health outcomes for women and their babies.

Competing interests: The BM/ has judged that there are no disqualifying financial ties to commercial companies. The authors declare no other interests. Further details of The BMJpolicy on financial interests is here: https://www.bmj.com/sites/default/files/attachments/resources/2016/03/16-current-bmj-education-coi-form.pdf.

Provenance and peer review: Not commissioned; externally peer reviewed.

We thank Rosanna Moss and Jane Brewin, who reviewed this editorial as patient and patient advocate, respectively.

1 Intensive Care National Audit and Research Centre. Covid-19 report. ICNARC covid-19 study case mix program database. Feb 2021. https://www.icnarc.org/Our-Audit/Audits/Cmp/Reports

2 CDC Covid data tracker. 20 Sep 2021. https://covid.cdc.gov/covid-data-tracker/\#pregnant-population

3 Takemoto MLS, Nakamura-Pereira M, Menezes MO, etal. Higher case fatality rate among obstetric patients with covid-19 in the second year of pandemic in Brazil: do new genetic variants play a role?medRxiv 2021;2021.05.06.21256651. [Preprint.] doi: 10.1101/2021.05.06.21256651

4 Mahajan NN, Pophalkar M, Patil S, etal. Pregnancy outcomes and maternal complications during the second wave of coronavirus disease 2019 (COVID-19) in India. Obstet Gynecol 2021. . doi: 10.1097/AOG.0000000000004529 pmid: 34233345

5 Brazil asks women to delay pregnancy over new coronavirus variant fears | Reuters 2021 Apr 16. https://www.reuters.com/world/americas/brazil-scrambles-secure-sedatives-hospitals-overwhelmed-by-covid-19-2021-04-16/

6 Abbas-Hanif A, Rezai H, Ahmed SF, Ahmed A. The impact of COVID-19 on pregnancy and therapeutic drug development. Br J Pharmacol 2021. doi: 10.1111/bph.15582 pmid: 34085281

7 Cole S, Coppola P, Kerwash E, Nooney J, Lam SP. Pharmacokinetic characterization to enable medicine use in pregnancy, the potential role of physiologically-based pharmacokinetic modeling: a regulatory perspective. CPT Pharmacometrics Syst Pharmacol 2020;9:547-9. doi: 10.1002/psp4.12551 pmid: 32741152

8 Adam MP, Polifka JE, Friedman JM. Evolving knowledge of the teratogenicity of medications in human pregnancy. Am J Med Genet C Semin Med Genet 2011;157C:175-82. doi: 10.1002/ajmg.c.30313 pmid: 21766440

9 Kilich E, Dada S, Francis MR, etal. Factors that influence vaccination decision-making among pregnant women: A systematic review and meta-analysis. PLoS One 2020;15:e0234827. doi: 10.1371/journal.pone.0234827 pmid: 32645112

10 Razai MS, Osama T, McKechnie DGJ, Majeed A. Covid-19 vaccine hesitancy among ethnic minority groupsBMJ 2021;372:n513. doi: 10.1136/bmj.n513 pmid: 33637577

11 Shimabukuro TT, Kim SY, Myers TR, etalCDC v-safe COVID-19 Pregnancy Registry Team. Preliminary findings of mRNA covid-19 vaccine safety in pregnant persons. N Engl / Med 2021;384:2273-82. doi: 10.1056/NEJMoa2104983 pmid: 33882218

12 UK Government. Covid-19 vaccine surveillance reports. 11 Aug 2021. https://www.gov.uk/government/publications/covid-19-vaccine-surveillance-report

13 Royal College of Obstetricians. Pregnant women eligible for the COVID-19 booster vaccine urged to take up offer. https://www.rcog.org.uk/en/news/pregnant-women-eligible-for-the-covid-19booster-vaccine-urged-to-take-up-offer/

14 International Coalition of Medicines Regulatory Authorities. Pregnancy and lactation workshop. 2021. http://icmra.info/drupal/en/covid-19/9february2021

15 Mullard A. 2020 FDA drug approvals. Nat Rev Drug Discov 2021;20:85-90. doi: 10.1038/d41573-021-00002-0 pmid: 33402709

16 Hyrich KL, Verstappen SMM. Biologic therapies and pregnancy: the story so far. Rheumatology (Oxford) 2014;53:1377-85. doi: 10.1093/rheumatology/ket409 pmid: 24352337 